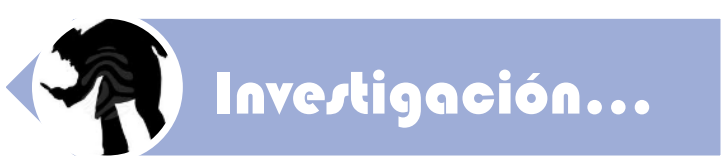

\title{
INFLUENCIA DEL INICIO DE RELACIONES SEXUALES EN COMPORTAMIENTOS VIOLENTOS EN ADOLESCENTES DE COLEGIOS PÚBLICOS DE LA LOCALIDAD DE SUBA
}

\author{
Carolina M. Velasco, Angie S. Mojica y Ana F. Nicola** \\ Tutor de la investigación: Juan Carlos González ${ }^{\dagger+}$
}

\section{RESUMEN}

Objetivo: Establecer relaciones entre conductas sexuales y comportamientos violentos de adolescentes escolarizados en colegios públicos de la localidad de Suba.

Metodología: Estudio observacional de corte transversal en 5871 adolescentes de 9 a 19 años de edad que estudiaban en colegios públicos ubicados en la localidad de Suba en el año 2010. Se realizó una encuesta en la que se indagó sobre varias conductas de riesgo para los adolescentes. Este artículo se enfoca solo en las variables sobre sexualidad y las relaciona con las de violencia.

Resultados: La prevalencia de relaciones sexuales es de $24 \%$. Se obtuvieron resultados significativos al comparar las variables "haber golpeado y haber sido golpeado" y "llevar algún tipo de arma para defenderse" con "haber tenido relaciones sexuales" (OR 2,73 IC $95 \%$ 2,41-3,09 y OR 4,04 IC $95 \%$ 3,444,76 , respectivamente), con "ha tenido relaciones sexuales sin querer" (OR 2,40 IC $95 \%$ de 1,98-2,92 y OR 3,39 IC $95 \% 2,72-4,24$, respectivamente) y con "ha tenido relaciones sexuales con más de una pareja en el último año" (OR 1,95 IC $95 \% 1,52-2,50$ y OR 2,13 IC $95 \% 1,64-2,77$, respectivamente).

Conclusiones: Los datos encontrados indican que hay una relación directa y significativa entre los comportamientos sexuales y la conducta violenta de la población estudiada.

Palabras claves: Adolescente, sexualidad, violencia, violencia de pareja, familia, factores de riesgo.

\section{ABSTRACT}

Objective: Establishing relations between sexual and violent behaviors of adolescents attending public schools from Suba locality.

Methodology: A cross-sectional observational study was carried out on a population of 5871 adolescents ranging in age from 9 to 26 years attending public schools at the urban area of Suba (Bogota) in 2010. A survey was made to study about several risk conducts for young people. Notwithstanding that, this article is just focused on variables related to sexuality and their relation to violence.

Results: Sexual relations prevalence is $24 \%$. Significant results were obtained when the variables "you have beaten or have been beaten" and, "you carry some kind of weapon to defend yourself" were compared with variables related to sexuality. The OR with "you have had sexual relations" was 2,73 (IC $95 \%$ 2,413,09 ) and, 4,04 (IC $95 \% 3,44-4,76$ ), respectively. Likewise the OR with "you have had sexual relations unintentionally" was 2,40 (IC $95 \%$ de 1,98-2,92) and, 3,39 (IC $95 \% 2,72-4,2$ ), respectively. And the OR with "you have had sexual relations with more than one couple in the last year" was 1,95 (IC $95 \% 1,52$ 2,50 ) and 2,13 (IC $95 \% 1,64-2,77$ ), respectively.

Conclusions: The results found indicate that there is a direct and significant relation between the sexual behavior and violence in the studied population.

Key words: Adolescent, sexuality, violence, intimate partner violence, family, risk factors.

** Estudiantes VII semestre 2013 (Investigación Social 2). Facultad de Medicina, FUJNC.

${ }^{+\dagger}$ MD. Especialista Medicina Familiar Integral, MSP. Director Departamento de Medicina Comunitaria juan.gonzalez@juanncorpas.edu.co 


\section{INTRODUCCIÓN}

La adolescencia es aceptada como una etapa del ciclo vital humano con personalidad propia, que se caracteriza por la magnitud y complejidad de las transformaciones que en esta se experimentan (1) por iniciarse la toma de decisiones (autonomía) y la búsqueda de pareja (aceptación de pares). Cronológicamente comienza alrededor de los 10 a 12 años y su final hacia los 19 a 20 años (2).

Debido al inicio abrupto de las experiencias dentro de las relaciones, se generan cambios en los comportamientos que pueden desencadenar conductas violentas para intentar resolver los problemas por sí mismos en busca del deseo de madurar y pasar a integrar el mundo adulto (3). Los adolescentes están comenzando sus primeras relaciones de pareja, probando y experimentando sentimientos encontrados y frustraciones nuevas, explorando emociones novedosas y agradables e intentando dar respuesta a miles de preguntas que tienen un nexo común: "¿es normal que...?" (4).

En esta etapa es difícil poder identificar conductas de riesgo, principalmente la violencia en las relaciones, ya que los adolescentes no perciben con claridad estas situaciones, puesto que se dan sus primeras experiencias afectivas y tienen un conocimiento irreal o idealizado sobre el amor (5).

Hoy los comportamientos agresivos en los adolescentes se han hecho mucho más visibles, se evidencian muy frecuentemente peleas, déficit en el control de impulsos, arranques de ira, amenazas, vandalismo, matoneo, crueldad y hasta muerte (6); en este sentido, el conocimiento de cómo surge y se modula el comportamiento agresivo es de vital importancia, debido a las consecuencias que la manifestación de dichas conductas tiene para los individuos y la sociedad. Estos comportamientos agresivos se empiezan a manifestar en la niñez temprana y adquieren su máxima expresión durante la adolescencia (7).

Aunque son variados los factores de riesgo que están detrás de los comportamientos agresivos fomentando su ocurrencia (7), la presente investigación pretende encontrar si existe relación directa entre las variables de los comportamientos sexuales y conductas violentas en adolescentes de colegios públicos de la localidad de Suba. Con esto se aspira aportar al entendimiento de los comportamientos violentos y que surjan posibilidades que permitan promover una mayor convivencia en nuestra sociedad.

\section{MATERIALES Y MÉTODOS}

Tipo de estudio: Se trata de un estudio analítico observacional, de corte o prevalencia.

Población: Se incluyeron adolescentes de 9 a 19 años de algunos colegios públicos de la localidad de Suba en Bogotá. Se solicitó permiso a los padres a través de las directivas del colegio.

Criterios de inclusión: Estar cursando algún año escolar, haber asistido el día de la aplicación de la encuesta y querer participar por elección propia.

Variables: Si bien se utilizó un cuestionario de 60 preguntas, para este estudio se tomaron variables sociodemográficas (edad, sexo y el estrato socioeconómico) y como variables referentes se preguntó si ha tenido o no relaciones sexuales, a qué edad las tuvo por primera vez y si usó algún método de protección en dicha situación (8). También se indagó si alguna vez ha tenido relaciones sexuales sin querer y si ha estado con más de una persona en el último año (9). Para las variables dependientes se realizaron las preguntas acerca de comportamientos de riesgo, en este caso, si golpeó o fue golpeado y si lleva algún tipo de arma para defenderse.

Recolección de la información: En el año 2010 se realizó esta encuesta en diferentes colegios públicos de la localidad de Suba a la población que cursa básica secundaria (10). La encuesta se llevó a cabo en las jornadas de la mañana y la tarde y se dispuso de 45 minutos para desarrollarla. Al iniciar se les aclaró a los estudiantes que la encuesta era con fines investigativos, que era anónima y que quien no quisiera participar estaba en todo su derecho de no hacerlo. Al cabo tres semanas se entregó el diagnóstico de riesgos a las directivas de cada colegio. 
Manejo estadístico: Se tabularon las respuestas en el programa EXCEL. Se analizaron las preguntas relacionadas con las variables de comportamientos sexuales y comportamientos violentos empleando el programa EPI INFO a partir del cual se obtuvieron los OR con sus límites y el valor de la prueba P.

\section{RESULTADOS}

De los 5871 adolescentes encuestados, el promedio de edad fue de 14 años, la edad mínima nueve años y máxima veintiséis años.

Tabla 1. Frecuencia de las características sociodemográficas y comportamientos de riesgo de la población de los adolescentes

\begin{tabular}{lrr}
\multicolumn{1}{c}{ VARIABLE } & No & \% \\
\hline Edad (años) & 1394 & 24 \\
De 10 a 12 & 3855 & 66 \\
De 13 a 16 & 615 & 10 \\
De 17 a 19 & 3303 & 58 \\
Estrato & 2171 & 38 \\
Uno y dos & 205 & 4 \\
Tres & & \\
Cuatro o más & 2908 & 49 \\
Sexo & 3007 & 51 \\
Masculino & 1426 & 24 \\
Femenino & 471 & 8 \\
Comportamientos sexuales & 376 & 7 \\
Ha tenido relaciones sexuales & & \\
Tener relaciones sexuales sin "amor" & \\
Ha tenido relaciones sexuales con más de una pareja en el & & \\
último año & 2165 & 38 \\
Comportamientos de riesgos de violencia & 729 & 13 \\
Ha sido golpeado (o ha golpeado) en el último año &
\end{tabular}

La edad promedio de inicio de vida sexual fue 13,6 años.

Tabla 2. Tabla de riesgo relativo de las variables de sexualidad en relación con los comportamientos violentos de adolescentes encuestados de colegios públicos en la localidad de Suba en el año 2010

\begin{tabular}{|c|c|c|c|c|c|c|c|c|c|c|c|c|c|c|c|c|}
\hline \multirow{3}{*}{$\begin{array}{l}\text { COMPORTAMIENTOS } \\
\text { RELACIONADOS CON LA } \\
\text { SEXUALIDAD }\end{array}$} & \multicolumn{8}{|c|}{ Golpeó y fue golpeado } & \multicolumn{8}{|c|}{ Lleva algún tipo de arma para defenderse } \\
\hline & \multicolumn{2}{|c|}{ Sí } & \multicolumn{2}{|c|}{ No } & \multirow{2}{*}{ OR } & \multirow{2}{*}{ LI } & \multirow{2}{*}{ LS } & \multirow{2}{*}{ Valor P } & \multicolumn{2}{|c|}{ sí } & \multicolumn{2}{|c|}{ No } & \multirow{2}{*}{ OR } & \multirow{2}{*}{ LI } & \multirow{2}{*}{ LS } & \multirow{2}{*}{ Valor P } \\
\hline & No & $\%$ & No & $\%$ & & & & & No & $\%$ & No & $\%$ & & & & \\
\hline Sí ha tenido relaciones sexuales & 819 & 59 & 572 & 41 & & & & & 368 & 27 & 1015 & 73 & & & & \\
\hline No ha tenido relaciones sexuales & 1450 & 34 & 2766 & 66 & & 1 & & & 346 & 25 & 3864 & 75 & & & & \\
\hline $\begin{array}{l}\text { Sí ha tenido relaciones sexuales } \\
\text { sin querer } \\
\text { No ha tenido relaciones sexuales } \\
\text { sin querer }\end{array}$ & $\begin{array}{r}271 \\
1764\end{array}$ & 37 & $\begin{array}{r}189 \\
2953\end{array}$ & $\begin{array}{l}41 \\
63\end{array}$ & 2,4 & 1,98 & 2,92 & 0,000000 & $\begin{array}{l}132 \\
501\end{array}$ & $\begin{array}{l}29 \\
11\end{array}$ & $\begin{array}{r}326 \\
4205\end{array}$ & $\begin{array}{l}71 \\
89\end{array}$ & 3,39 & 2,72 & 4,24 & 0,000000 \\
\hline $\begin{array}{l}\text { Si ha tenido relaciones sexuales } \\
\text { con más de una pareja en el } \\
\text { último año } \\
\text { No ha tenido relaciones sexuales } \\
\text { con más de una pareja en el } \\
\text { último año }\end{array}$ & 548 & 67 & 122 & 33 & 1,95 & 1,52 & 2,5 & 0,000001 & 222 & 21 & 850 & 64 & 2,13 & 1,64 & 2,77 & 0,000000 \\
\hline
\end{tabular}

\section{DISCUSIÓN}

El sesgo más probable en esta investigación es el de información dado que los adolescentes podrían omitir o alterar los datos suministrados en las encuestas. Se intentó disminuir lo anterior aclarando que ellos podían participar de manera voluntaria, no estar obligados a responder todas las preguntas si así lo querían y la garantía de la confidencialidad de las respuestas dado que no tenían que ser marcadas con su nombre. 
En este estudio se encontró que el inicio de las relaciones coitales es antes de los 14 años, dato similar al del estudio de Profamilia (12). Según los datos hallados en el presente estudio (tabla 2) se evidencia una relación entre los comportamientos violentos y las relaciones sexuales en los adolescentes, planteándose de esa manera que estas asociaciones se pudieran interpretar como actos de control y comportamientos agresivos que ocurren en una relación romántica entre parejas que no conviven, con el objeto de controlar o dominar a la persona física, sexual o psicológicamente (13). Aunque no se indagó sobre variables relacionadas con lo anterior (control o celos) es importante considerar el inicio de una relación de pareja como un factor influyente para las conductas violentas de los adolescentes, puesto que la característica principal de este tipo de violencia (de pareja) son las conductas de control que pueden incluir prohibir relaciones con amigos del sexo opuesto, llamadas y búsqueda frecuente para averiguar dónde se encuentra, con quién está, qué está haciendo, exigir que debe pasar el mayor tiempo con él o con ella o también imponer los criterios a la hora de vestir (14).

Este tipo violencia puede ser acompañado por el abuso verbal y emocional, por ejemplo, las desvalorizaciones, burlas, insultos, menosprecios, amenazar con hacer daño a alguien de su familia o a la misma persona, si no hace lo que la otra persona quiere. Además, se puede presentar violencia física como empujar, morder, golpear, cachetear, patear, halar el pelo o estrangular, con el objetivo de hacer daño y someter a su pareja (15). La salud mental de una población puede determinar un aumento de conductas no saludables, en este caso los adolescentes (16).

Otra explicación de este tipo de asociación se puede presentar por parte del abuso sexual como contacto físico no deseado, obligar a tener relaciones sexuales, no permitir el uso de un método anticonceptivo, obligar hacer actos sexuales indeseados (17). Puede suceder que las personas que hayan sido víctimas de abuso sexual pueden repetir conductas agresivas en contra de otros con el fin de generar alivio y desahogo ante los sentimientos guardados de asco, rechazo, horror, afán de venganza, miedo e inseguridad, que en ocasiones pueden conllevar a un efecto de hipersexualidad que lo predisponen a convertirse en un futuro abusador (18).

Para los jóvenes, el inicio de una vida sexual activa implica, ante todo, el aprendizaje de habilidades sociales propias de su contexto. Por ejemplo, aprenden cuándo, cómo y con quién, pueden tener relaciones sexuales. Significa, asimismo, que mientras más habilidades sexuales se tengan, mayor será el prestigio y el poder que se adquiera entre sus compañeros (17) lo que genera un riesgo de tener relaciones sexuales solamente en busca de querer la aceptación social.

En cuanto a la conducta de tener más de una pareja sexual en el último año y el porte de armas se observa una mayor relación con un OR de 2,13 (IC95 \% 1,64-2,77) frente al golpear y ser golpeado (OR 1,95 - IC95 \% 1,52-2.5). En torno a la relación entre llevar armas y relaciones sexuales, surge la posibilidad de pertenecer a pandillas (violencia y criminalidad) y la presión de una cultura de consumo donde se promueve la posibilidad de acercamiento a las pandillas para cubrir dos o más de las necesidades del momento, a saber, identidad y protección (propia y para la familia), placer inmediato y ausencia de límites para la acción (19). Esto permite al adolescente ser aceptado como igual entre sus pares, ser protegido de las fuerzas externas que amenazan su integridad y tener cubiertas otras necesidades a través del estilo de vida de las pandillas (20).

En tal sentido el adolescente considera a la pandilla como un espacio propio, sin intervención adulta, con expresiones propias de esa etapa de la vida y sin límites (21). La "grupalidad" está al servicio de algunas de las conductas desviadas en las que se implican tales colectivos, en concreto la violencia, procurando que se mantengan en el margen de las consecuencias simbólicas esperadas y evite caer en el de las consecuencias reales temidas (22). La "grupalidad" permite la reconstrucción categorial de la realidad, señala las víctimas, elabora los argumentos que justifican la animadversión y el ataque contra las mismas, proporciona apoyo y protección materiales frente a la violencia de otros, soporta y valida el "ideal" que, a su vez, habilita estándares de autoevaluación y autorrealización (23). 
El objetivo de las pandillas no es solo robar o especializarse en cometer actos ilícitos, también está el expandir el desenfreno sexual, el consumo de alcohol, drogas, tráfico de armas y matar (21). Según la tabla 2 se evidencia que por cada adolescente que no ha tenido relaciones sexuales y porta armas para defenderse hay 4,04 que sí han tenido relaciones sexuales y portan armas para defenderse, lo que evidencia que hay un riesgo 4 veces más de tener comportamientos violentos. El porte de armas podría estar relacionado con vinculación a pandillas o a grupos urbanos similares, que facilitan el acceso a armas y a conductas sexuales por aceptación de sus pares.

La adolescencia constituye una etapa de la vida diferenciable, con sus propias características y necesidades en salud. Las conductas tomadas por los adolescentes son su eje para desarrollar fortalezas (24). Esta investigación más exploratoria que otra cosa, ha abordado unas asociaciones que deberán indagarse más, máxime ante la cercanía del postconflicto, que implica acercarnos a entender para estar en capacidad de proponer.

\section{REFERENCIAS}

(1) Santiago M, López J, Navarro R. Adolescencia. Aspectos generales y atención a la salud. Rev Cubana Med Gen Integr. 2006; 22(1):9-11

(2) González J, De la Hoz F. Relaciones entre los comportamientos de riesgo psicosociales y la familia en adolescentes de Suba, Bogotá. Rev. Salud pública. 2011;(3):67-78.

(3) Castaño E. "Te quiero... (solo para mi)" relaciones adolescentes de control. TABANQUE Revista pedagógica, Universidad de Valladolid 2010; 23: 45-68.

(4) Copp J, Giordano P, Longmore M, Manning W. Stay-or-Leave decision making in nonviolent and violent dating relationships. Violence and Victims. 2010; 30(4): 581-599.

(5) Stephenson K, Meston C. When are sexual difficulties distressing for women? The selective protective value of intimate relationships. J Sex Med. 2010; 7(11): 3683-3694.

(6) Díaz JL, De la Peña F, Suárez JA, Palacios L. Perspectiva actual de la violencia juvenil. MedUNAB. 2004; 7:115-242.

(7) Andreu J, Peña M. Agresividad reactiva y proactiva en adolescentes: efecto de los factores individuales y sociocontextuales. Universidad complutense de Madrid facultad de psicología.2012.

(8) Correa E, Mancillas C, Pachecho W, Carreño C, Castañeda J, Iturbe M, et al. Psicología iberoamericana. 2006. 14(2): 163.

(9) Matla E, Fuentes Y. Estado de salud y disfunción familiar en pacientes con hipertensión arterial en primer nivel de atención. México, 2011.

(10) Alarcón R, Coello J, Cabrera J y Monier G. Factores que influyen en el embarazo en la adolescencia. Rev Cubana Enfermer. 2009. 25(1-29).

(11) Ministerio de salud y protección social. Resolución 1841 de 2013.

(12) ISS - Profamilia - Instituto Nacional de Cancerología, Encuesta sobre CAP en adolescentes, 1995.

(13) Grover A. Risky lifestyles and dating violence: A theoretical test of violent victimization. Journal of Criminal Justice, 2004; 32: 171-180.

(14) Omidi R, Heidari K, Davari H, Espanani M, Poursalehi M. The Relationships between Environmental Factors and Violent Behaviors in Adolescent Students of Isfahan, Iran. Int J Prev Med. 2014; 5 (2):S97-S101.

(15) Martínez J. Violencia en el noviazgo: Un programa de intervención conductual en adolescentes escolarizados. Repositorio Pontificia Universidad Javeriana. Facultad de Psicología. Maestría en Psicología Clínica, 2014.

(16) Corona F, Peralta E. Prevención de conductas de riesgo. rev. med. clin. Condes. 2011; 22(1): 68-75.

(17) Sánchez M. Entre la experiencia, el amor y el riesgo. Repositorio Universidad Nacional de Colombia. $2003 ; 99-111$.

(18) Jiménez S, Urbina C. Prevención de conductas delictivas en estudiantes de colegios públicos del municipio de Sopó. Repositorio Universidad de la Sabana, 2006.

(19) Soria B. Informe de Consultoría sobre definición y categorización de pandillas - Ecuador. Quito, mayo de 2007.

(20) Moncaleano G. Incidencia de la violencia de pandillas en la seguridad nacional de los países del hemisferio." Universidad del Salvador, mayo de 2006.

(21) Domínguez N. De cuerpos urbanos violentados. Revista jóvenes México: Centro de Investigaciones y Estudios sobre Juventud, 2001.

(22) Scandroglio B, López J, Martínez J, San José M, Martín M, Martín A. La conducta violenta en grupos juveniles: características descriptivas. Revista de estudios de juventud, 2003 62, 151-158.

(23) San José M, Scandroglio B, López J, Martín M, Martínez J, Martín A. La teoría del comportamiento planificado: un modelo explicativo en el estudio de la violencia grupal juvenil. Encuentros en psicología social. 2003; 1(2): $134-140$.

(24) Frankowski BL, Leader IC, Duncan PM. Strength-Based Interviewing. Adolesc. Med. 2009; 20: 22-40.

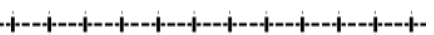

Carta Comunitaria. Vol. 24. Número 138. Mayo - Junio 2016 